Pacific

Journal of

Mathematics

OPERATOR SPACE CHARACTERIZATIONS OF C*-ALGEBRAS AND TERNARY RINGS

Matthew Neal and Bernard Russo 


\title{
OPERATOR SPACE CHARACTERIZATIONS OF C*-ALGEBRAS AND TERNARY RINGS
}

\author{
Matthew Neal and Bernard Russo
}

\begin{abstract}
We prove that an operator space is completely isometric to a ternary ring of operators if and only if the open unit balls of all of its matrix spaces are bounded symmetric domains. From this we obtain an operator space characterization of C*-algebras.
\end{abstract}

\section{Introduction.}

In the category of operator spaces, that is, subspaces of the bounded linear operators $B(H)$ on a complex Hilbert space $H$ together with the induced matricial operator norm structure, objects are equivalent if they are completely isometric, i.e., if there is a linear isomorphism between the spaces which preserves this matricial norm structure. Since operator algebras, that is, subalgebras of $B(H)$, are motivating examples for much of operator space theory, it is natural to ask if one can characterize which operator spaces are operator algebras. One satisfying answer was given by Blecher, Ruan and Sinclair in [10], where it was shown that among operator spaces $A$ with a (unital but not necessarily associative) Banach algebra product, those which are completely isometric to operator algebras are precisely the ones whose multiplication is completely contractive with respect to the Haagerup norm on $A \otimes A$. (For a completely bounded version of this result, see [7].)

A natural object to characterize in this context are the so called ternary rings of operators (TRO's). These are subspaces of $B(H)$ which are closed under the ternary product $x y^{*} z$. This class includes $\mathrm{C}^{*}$-algebras. TRO's, like $\mathrm{C}^{*}$-algebras, carry a natural operator space structure. In fact, every TRO is (completely) isometric to a corner $p A(1-p)$ of a $\mathrm{C}^{*}$-algebra $A$. TRO's are important because, as shown by Ruan [35], the injectives in the category of operator spaces are TRO's (corners of injective $\mathrm{C}^{*}$-algebras) and not, in general, operator algebras. (For the dual version of this result see [15].) Injective envelopes of operator systems and of operator spaces ([23] and [35]) have proven to be important tools, see for example [9]. The characterization of TRO's among operator spaces is the subject of this paper. (See Theorem 5.3.)

Closely related to TRO's are the so called JC*-triples, norm closed subspaces of $B(H)$ which are closed under the triple product $\left(x y^{*} z+z y^{*} x\right) / 2$. 
These generalize the class of TRO's and have the property, as shown by Harris in [25], that isometries coincide with algebraic isomorphisms. It is not hard to see this implies that the algebraic isomorphisms in the class of TRO's are complete isometries, since for each TRO $A, M_{n}(A)$ is a $\mathrm{JC}^{*}$-triple. (For the converse of this, see [24, Proposition 2.1].) As a consequence, if an operator space $X$ is completely isometric to a TRO, then the induced ternary product on $X$ is unique, i.e., independent of the TRO.

Building on the pioneering work of Arveson ([3] and [4]) on noncommutative analogs of the Choquet and Shilov boundaries, Hamana (see [24]) proved that every operator space $A$ has a unique enveloping TRO $\mathcal{T}(A)$ which is an invariant of complete isometry and has the property that for any TRO $B$ generated by a realization of $A$, there exists a homomorphism of $B$ onto $\mathcal{T}(A)$. The space $\mathcal{T}(A)$ is also called the Hilbert $\mathrm{C}^{*}$-envelope of $A$. The work in $[8]$ suggests that the Hilbert $\mathrm{C}^{*}$-envelope is an appropriate noncommutative generalization to operator spaces of the classical theory of Shilov boundary of function spaces.

It is also true that a commutative TRO $\left(x y^{*} z=z y^{*} x\right)$ is an associative $\mathrm{JC}^{*}$-triple and hence by [19, Theorem 2], is isometric (actually completely isometric) to a complex $C_{\mathrm{hom}}$-space, that is, the space of weak*-continuous functions on the set of extreme points of the unit ball of the dual of a Banach space which are homogeneous with respect to the natural action of the circle group, see [19]. Hence, if one views operator spaces as noncommutative Banach spaces, and $\mathrm{C}^{*}$-algebras as noncommutative $C(\Omega)$ 's, then TRO's and $\mathrm{JC}^{*}$-triples can be viewed as noncommutative $C_{\mathrm{hom}}$-spaces.

As noted above, injective operator spaces, i.e., those which are the range of a completely contractive projection on some $B(H)$, are completely isometrically TRO's; the so called mixed injective operator spaces, those which are the range of a contrative projection on some $B(H)$, are isometrically $\mathrm{JC}^{*}$-triples. The operator space classification of mixed injectives was begun by the authors in [32] and [33] and is ongoing.

Relevant to this paper is another property shared by all $\mathrm{JC}^{*}$-triples (and hence all TRO's). For any Banach space $X$, we denote by $X_{0}$ its open unit ball: $\{x \in X:\|x\|<1\}$. The open unit ball of every $\mathrm{JC}^{*}$-triple is a bounded symmetric domain. This is equivalent to saying that it has a transitive group of biholomorphic automorphisms. It was shown by Koecher in finite dimensions (see [31]) and Kaup [28] in the general case that this is a defining property for the slightly larger class of JB*-triples. The only illustrative basic examples of $\mathrm{JB}^{*}$-triples which are not $\mathrm{JC}^{*}$-triples are the space $H_{3}(\mathcal{O})$ of $3 \times 3$ Hermitian matrices over the octonians and a certain subtriple of $H_{3}(\mathcal{O})$. These are called exceptional triples, and they cannot be represented as a $\mathrm{JC}^{*}$-triple. This holomorphic characterization has been useful as it gives an elegant proof, due to Kaup [29], that the range of a contractive projection on a $\mathrm{JB}^{*}$-triple is isometric to another $\mathrm{JB}^{*}$-triple. The same 
statement holds for $\mathrm{JC}^{*}$-triples, as proven earlier by Friedman and Russo in $[\mathbf{2 1}]$. Youngson proved in [38] that the range of a completely contractive projection on a $\mathrm{C}^{*}$-algebra is completely isometric to a TRO. These results, as well as those of [2] and [17], are rooted in the fundamental result of Choi-Effros $[12]$ for completely positive projections on $\mathrm{C}^{*}$-algebras and the classical result $([\mathbf{3 0}]$ and $[\mathbf{1 8}$, Theorem 5]) that the range of a contractive projection on $C(\Omega)$ is isometric to a $C_{\sigma^{-}}$-space, hence a $C_{\mathrm{hom}}$-space.

Motivated by this characterization for JB*-triples, we will give a holomorphic characterization of TRO's up to complete isometry. We will prove in Theorem 5.3 that an operator space $A$ is completely isometric to a TRO if and only if the open unit balls $M_{n}(A)_{0}$ are bounded symmetric domains for all $n \geq 2$. As a consequence, we obtain in Theorem 5.7 a holomorphic operator space characterization of $\mathrm{C}^{*}$-algebras as well. It should be mentioned that Upmeier (for the category of Banach spaces) in [37] and El AminCampoy-Palacios (for the category of Banach algebras) in [1], gave different but still holomorphic characterizations of $\mathrm{C}^{*}$-algebras up to isometry. We note in passing that injective operator spaces satisfy the hypothesis of Theorem 5.3, so we obtain that they are (completely isometrically) TRO's based on deep results about $\mathrm{JB}^{*}$-triples rather than the deep result of Choi-Effros. (See Corollaries 5.5 and 5.6.)

We now describe the organization of this paper. Section 2 contains the necessary background and some preliminary results on contractive projections. In Section 3, three auxiliary ternary products are introduced and are shown to yield the original $\mathrm{JB}^{*}$-triple product upon symmetrization. Section 4 is devoted to proving that these three ternary products all coincide. Section 5 contains the statement and proof of the main result and its consequences.

\section{Preliminaries.}

An operator space will be defined as a normed space $A$ together with a linearly isometric representation as a subspace of some $B(H)$. This gives $A$ a family of operator norms $\|\cdot\|_{n}$ on $M_{n}(A) \subset B\left(H^{n}\right)$. As proved in [34], an operator space can also be defined abstractly as a normed space $A$ having a norm on $M_{n}(A)(n \geq 2)$ satisfying certain properties. Each such family of norms is regarded as a "quantization" of the underlying Banach space. These properties give rise to an isometric representation of the operator space as a subspace of $\mathrm{B}(\mathrm{H})$ where the natural amplification maps preserve the matricial norm structure. This is analagous to (and generalizes) the way an abstract Banach space $B$ can be isometrically embedded as a subspace of $C(\Omega)$. The resulting operator space structure in this case is called $M I N(B)$ and is seen as a commutative quantization of $B$. 
Two operator spaces $A$ and $B$ are $\boldsymbol{n}$-isometric if there exists an isometry $\phi$ from $A$ onto $B$ such that the amplification mapping $\phi_{n}: M_{n}(A) \rightarrow M_{n}(B)$ defined by $\phi\left(\left[a_{i j}\right]\right)=\left[\phi\left(a_{i j}\right)\right]$ is an isometry. $A$ and $B$ are completely isometric if there exists a mapping $\phi$ from $A$ onto $B$ which is an $n$-isometry for all $n$. For other basic results about operator spaces, see [16].

The following definition is a Hilbert space-free generalization of the TRO's mentioned in the introduction:

Definition 2.1 (Zettl [39]). A $\mathrm{C}^{*}$-ternary ring is a Banach space $A$ with ternary product $[x, y, z]: A \times A \times A \rightarrow A$ which is linear in the outer variables, conjugate linear in the middle variable, is associative:

$$
[a b[c d e]]=[a[d c b] e]=[a b[c d e]],
$$

and satisfies $\|[x y z]\| \leq\|x\|\|y\|\|z\|$ and $\|[x x x]\|=\|x\|^{3}$.

$\mathrm{A}$ TRO is a $\mathrm{C}^{*}$-ternary ring under any of the products $[x y z]_{\lambda}=\lambda x y^{*} z$, for any complex number $\lambda$ with $|\lambda|=1$.

A linear map $\varphi$ between $\mathrm{C}^{*}$-ternary rings is a homomorphism if $\varphi([x y z])$ $=[\varphi(x), \varphi(y), \varphi(z)]$ and an anti-homomorphism if $\varphi([x y z])=-[\varphi(x)$, $\varphi(y), \varphi(z)]$.

The following is a Gelfand-Naimark representation theorem for $\mathrm{C}^{*}$-ternary rings:

Theorem 2.2 ([39]). For any $\mathrm{C}^{*}$-ternary ring $A, A=A_{1} \oplus A_{-1}$, where $A_{1}$ and $A_{-1}$ are sub-C $\mathrm{C}^{*}$-ternary rings, $A_{1}$ is isometrically isomorphic to a TRO $B_{1}$ and $A_{-1}$ is isometrically anti-isomorphic to a TRO $B_{-1}$.

It follows that $A_{-1}=0$ if and only if $A$ is ternary isomorphic to a TRO. In Theorem 5.3, we shall show that under suitable assumptions on an operator space $A$, it becomes a $\mathrm{C}^{*}$-ternary ring with $A_{-1}=0$ and the above ternary isomorphism is a complete isometry from $A$ with its original operator space structure to a TRO with its natural operator space structure.

An immediate consequence of our proof of Theorem 5.3 is an answer to a question posed by Zettl $\left[39\right.$, p. 136]: For a $\mathrm{C}^{*}$-ternary ring $A, A_{-1}=0$ if and only if $A$ is a $\mathrm{JB}^{*}$-triple (see the next definition) under the triple product

$$
\{a b c\}=\frac{1}{2}([a b c]+[c b a]) .
$$

The following definition generalizes the $\mathrm{JC}^{*}$-triples defined in the introduction:

Definition $2.3([28])$. A $\mathbf{J B}^{*}$-triple is a Banach space $A$ with a product $D(x, y) z=\left\{\begin{array}{lll}x & y & z\end{array}\right\}$ which is linear in the outer variables, conjugate linear in the middle variable, is commutative: $\left\{\begin{array}{lll}x & y & z\end{array}\right\}=\left\{\begin{array}{lll}z & y & x\end{array}\right\}$, satisfies an 
associativity condition:

$$
[D(x, y), D(a, b)]=D\left(\left\{\begin{array}{lll}
x & y & a
\end{array}\right\}, b\right)-D\left(a,\left\{\begin{array}{lll}
b & x & y
\end{array}\right\}\right)
$$

and has the topological properties that:

(i) $\|D(x, x)\|=\|x\|^{2}$,

(ii) $D(x, x)$ is Hermitian (in the sense that $\left\|e^{i t D(x, x)}\right\|=1$ ) and has positive spectrum in the Banach algebra $B(A)$.

We abbreviate $D(x, x)$ to $D(x)$.

As noted in the introduction, $\mathrm{JC}^{*}$-triples (and hence TRO's and $\mathrm{C}^{*}$ algebras) are examples of $\mathrm{JB}^{*}$-triples. Other examples include any Hilbert space, and the spaces of symmetric and anti-symmetric elements of $B(H)$ under a transpose map defined by a conjugation.

If one ignores the norm and the topological properties in Definition 2.3, the algebraic structure which results, called a Jordan triple system, or Jordan pair, has a life of its own, [31]. Note that (1) can be written as

$$
\{x, y,\{a b z\}\}-\{a, b,\{x y z\}\}=\{\{x y a\}, b, z\}-\{a,\{y x b\}, z\} .
$$

For easy reference we record here two identities for Jordan triple systems which can be derived from (1) ([31, JP8, JP16]).

$$
\begin{gathered}
2 D(x,\{y x z\})=D(\{x y x\}, z)+D(\{x z x\}, y) \\
\{\{x y a\}, b, z\}-\{a,\{y x b\}, z\}=\{x,\{b a y\}, z\}-\{\{a b x\}, y, z\} .
\end{gathered}
$$

We will now list some facts about $\mathrm{JB}^{*}$-triples that are relevant to our paper. A survey of the basic theory can be found in [36]. As proved by Kaup [28], JB*-triples are in 1-1 isometric correspondence with Banach spaces whose open unit ball is a bounded symmetric domain. The triple product here arises from the Lie algebra of the group of biholomorphic automorphisms. This Lie algebra is the space of complete vector fields on the open unit ball and consists of certain polynomials of degree at most 2 . The quadratic term in each of these polynomials is determined by the constant term. For a bounded symmetric domain, the constant terms which occur exhaust $A$. Thus, linearizing the quadratic term for every element $a \in A$ leads to a triple product on $A$.

It is this correspondence which motivates the study of the more general JB*-triples. Indeed, the proofs of two important facts follow naturally from the holomorphic point of view [29]. Firstly, the isometries between $\mathrm{JB}^{*}$-triples are precisely the algebraic isomorphisms. From this follows the important fact, used several times in this paper, that, unlike the case for binary products, the triple product of a JB*-triple is unique. Secondly, the 
range of a contractive projection $P$ on a $\mathrm{JB}^{*}$-triple $Z$ is isometric to a $\mathrm{JB}^{*}$ triple. More precisely, $P(Z)$ is a $\mathrm{JB}^{*}$-triple under the norm and linear operations it inherits from $Z$ and the triple product $\{x y z\}_{P(Z)}:=P\left(\{x y z\}_{Z}\right)$, for $x, y, z \in P(Z)$.

In the context of $\mathrm{JC}^{*}$-triples, these facts were proven by functional analytic methods in $[\mathbf{2 5}]$ and $[\mathbf{2 1}]$ respectively. These facts show that JB*-triples are a natural category in which to study isometries and contractive projections. Recently, in [13] the authors with C.-H. Chu have shown that w*continuous contractive projections on dual $\mathrm{JB}^{*}$-triples (called $\mathrm{JBW}^{*}$-triples) preserve the Jordan triple generalization of the Murray-von-Neumann type decomposition established in [26] and [27]. Two other properties of contractive projections were used in that work and will be needed in the present paper. They consist of two conditional expectation formulas for contractive projections on $\mathrm{JC}^{*}$-triples $([\mathbf{2 0}$, Corollary 1$])$, namely

$$
P\{P x, P y, P z\}=P\{P x, P y, z\}=P\{P x, y, P z\} ;
$$

and the fact that the range of a bicontractive projection on a $\mathrm{JC}^{*}$-triple is a subtriple [20, Proposition 1]. Recall that a projection $P$ is said to be bicontractive if $\|P\| \leq 1$ and $\|I-P\| \leq 1$.

Let $A$ be a JB*-triple. For any $a \in A$, there is a triple functional calculus, that is, a triple isomorphism of the closed subtriple $C(a)$ generated by $a$ onto the commutative $\mathrm{C}^{*}$-algebra $C_{0}(\operatorname{Sp} D(a, a) \cup\{0\})$ of continuous functions vanishing at zero, with the triple product $f \bar{g} h$. Any $\mathrm{JBW}^{*}$-triple (defined in the previous paragraph) has the propertly that it is the norm closure of the linear span of its tripotents, that is, elements $e$ with $e=\{e e e\}$. A unitary tripotent is a tripotent $v$ such that $D(v, v)=I d$. For a $\mathrm{C}^{*}$-algebra, tripotents are the partial isometries and for unital $\mathrm{C}^{*}$-algebras, unitary tripotents are precisely the unitaries. For tripotents $u$ and $v$, algebraic orthogonality, i.e., $D(u, v)=0$, coincides with Banach space othogonality: $\|u \pm v\|=1$. For $a$ and $b$ in $A$, we will denote the property $D(a, b)=0$ by $a \perp b$.

As proved in [14], the second dual $A^{* *}$ of a $\mathrm{JB}^{*}$-triple $A$ is a $\mathrm{JBW}^{*}$ triple containing $A$ as a subtriple. Multiplication in a $\mathrm{JBW}^{*}$-triple is norm continuous and, as proved in [5], separately $\mathrm{w}^{*}$-continuous.

We close this section of preliminaries with an elementary proposition showing that certain concrete projections are contractive.

Proposition 2.4. Let $A$ be an operator space in $B(H)$.

(a) Define a projection $\mathrm{P}$ on $M_{2}(A)$ by

$$
P\left(\left[\begin{array}{ll}
a & b \\
c & d
\end{array}\right]\right)=\frac{1}{2}\left[\begin{array}{cc}
a+b & a+b \\
0 & 0
\end{array}\right] .
$$

Then $\|P\| \leq 1$. Moreover, the restriction of $P$ to $\left\{\left[\begin{array}{ll}a & b \\ 0 & 0\end{array}\right]: a, b \in A\right\}$ is bicontractive. 
(b) Let $P_{11}: M_{2}(A) \rightarrow M_{2}(A)$ be the map $\left[\begin{array}{ll}a_{11} & a_{12} \\ a_{21} & a_{22}\end{array}\right] \mapsto\left[\begin{array}{cc}a_{11} & 0 \\ 0 & 0\end{array}\right]$, and similarly for $P_{12}, P_{21}, P_{22}$. Then $P_{i j}$ is contractive and $P_{11}+P_{21}$, $P_{11}+P_{12}$, and $P_{11}+P_{22}$ are bicontractive. More generally, the $P_{i j}:$ $M_{n}(A) \rightarrow M_{n}(A)$ are contractive and for any subset $S \subset\{1,2, \ldots, n\}$, $\sum_{i \in S} \sum_{j=1}^{n} P_{i j}$ and $\sum_{j \in S} \sum_{i=1}^{n} P_{i j}$ are bicontractive.

(c) The projections $P: M_{2}(A) \rightarrow M_{2}(A)$ and $Q: P\left(M_{2}(A)\right) \rightarrow$ $P\left(M_{2}(A)\right)$ defined by

$$
P\left(\left[\begin{array}{ll}
a & b \\
c & d
\end{array}\right]\right)=\frac{1}{2}\left[\begin{array}{ll}
a+d & b+c \\
b+c & a+d
\end{array}\right]
$$

and

$$
Q\left(\left[\begin{array}{ll}
a & b \\
b & a
\end{array}\right]\right)=\frac{1}{2}\left[\begin{array}{ll}
a+b & a+b \\
a+b & a+b
\end{array}\right]
$$

are bicontractive.

Proof. We omit the proofs of (a) and (b). To prove (c), since for example $I-P=(I-(2 P-I)) / 2$ and $P=(I+(2 P-I)) / 2$, it suffices to show that $2 P-I$ and $2 Q-I$ are contractive. But

$$
(2 P-I)\left(\left[\begin{array}{ll}
a & b \\
c & d
\end{array}\right]\right)=\left[\begin{array}{ll}
d & c \\
b & a
\end{array}\right]=\left[\begin{array}{ll}
0 & 1 \\
1 & 0
\end{array}\right]\left[\begin{array}{ll}
a & b \\
c & d
\end{array}\right]\left[\begin{array}{ll}
0 & 1 \\
1 & 0
\end{array}\right],
$$

and

$$
(2 Q-I)\left(\left[\begin{array}{ll}
a & b \\
b & a
\end{array}\right]\right)=\left[\begin{array}{ll}
b & a \\
a & b
\end{array}\right]=\left[\begin{array}{ll}
0 & 1 \\
1 & 0
\end{array}\right]\left[\begin{array}{ll}
a & b \\
b & a
\end{array}\right]
$$

\section{Additivity of the ternary products.}

Throughout this section, $A \subset B(H)$ will be an operator space such that the open unit ball $M_{2}(A)_{0}$ is a bounded symmetric domain. Let $\{\cdot, \cdot, \cdot\}_{M_{2}(A)}$ denote the associated $\mathrm{JB}^{*}$-triple product on $M_{2}(A)$. Note that although $M_{2}(A)$ inherits the norm and linear structure of $M_{2}(B(H))=B(H \oplus H)$, its triple product $\{\cdots\}_{M_{2}(A)}$ in general differs from the concrete triple product $\left(X Y^{*} Z+Z Y^{*} X\right) / 2$ of $B(H \oplus H)$. In fact, the results of this section would become trivial if these two triple products were the same.

By properties of contractive projections and the uniqueness of the triple product, $A$, being linearly isometric to $P_{i j}\left(M_{2}(A)\right)$ becomes a JB*-triple whose triple product $\{x y z\}_{A}$ is given, for example, by

$$
\left[\begin{array}{cc}
\{x y z\}_{A} & 0 \\
0 & 0
\end{array}\right]=P_{11}\left(\left\{\left[\begin{array}{ll}
x & 0 \\
0 & 0
\end{array}\right]\left[\begin{array}{ll}
y & 0 \\
0 & 0
\end{array}\right]\left[\begin{array}{ll}
z & 0 \\
0 & 0
\end{array}\right]\right\}_{M_{2}(A)}\right),
$$


and similarly using the other $P_{i j}$. Usually we shall justuse the notation $\{\cdots\}$ for either of the triple products $\{x y z\}_{A}$ and $\{\cdot, \cdot, \cdot\}_{M_{2}(A)}$. Lemma 3.6 shows that the projection $P_{11}$ could be removed in this definition.

We assume $A$ is as above and proceed to define (in Definition 3.7) three auxiliary ternary products, denoted $[\cdot, \cdot, \cdot],(\cdot, \cdot, \cdot)$, and $\langle\cdot, \cdot, \cdot\rangle$ and show their relation to $\{\cdot, \cdot, \cdot\}$. We begin with a sequence of lemmas which establish some properties of the terms in the following identity, where $a, b, c \in A$ :

$$
\begin{aligned}
\left\{\left[\begin{array}{ll}
a & a \\
0 & 0
\end{array}\right]\left[\begin{array}{ll}
0 & b \\
0 & 0
\end{array}\right]\left[\begin{array}{ll}
c & c \\
0 & 0
\end{array}\right]\right\}= & \left\{\left[\begin{array}{ll}
a & 0 \\
0 & 0
\end{array}\right]\left[\begin{array}{ll}
0 & b \\
0 & 0
\end{array}\right]\left[\begin{array}{ll}
c & 0 \\
0 & 0
\end{array}\right]\right\} \\
& +\left\{\left[\begin{array}{ll}
a & 0 \\
0 & 0
\end{array}\right]\left[\begin{array}{ll}
0 & b \\
0 & 0
\end{array}\right]\left[\begin{array}{ll}
0 & c \\
0 & 0
\end{array}\right]\right\} \\
& +\left\{\left[\begin{array}{ll}
0 & a \\
0 & 0
\end{array}\right]\left[\begin{array}{ll}
0 & b \\
0 & 0
\end{array}\right]\left[\begin{array}{ll}
0 & c \\
0 & 0
\end{array}\right]\right\} \\
& +\left\{\left[\begin{array}{ll}
0 & a \\
0 & 0
\end{array}\right]\left[\begin{array}{ll}
0 & b \\
0 & 0
\end{array}\right]\left[\begin{array}{ll}
c & 0 \\
0 & 0
\end{array}\right]\right\} .
\end{aligned}
$$

It will be shown in Lemma 3.2 that the left side of (6) has the form

$$
\left[\begin{array}{ll}
x & y \\
z & w
\end{array}\right]
$$

where $(x+y) / 2=\{a b c\}$. In Lemmas 3.4-3.6, each term on the right side of (6) will be analyzed.

Remark 3.1. The space

$$
\widetilde{A}=\left\{\widetilde{a}=\left[\begin{array}{ll}
a & a \\
0 & 0
\end{array}\right]: a \in A\right\}
$$

with the triple product

$$
\{\widetilde{a} \widetilde{b}\}_{\widetilde{A}}:=\left[\begin{array}{cc}
2\{a b c\} & 2\{a b c\} \\
0 & 0
\end{array}\right]
$$

and the norm of $M_{2}(A)$, is a $\mathrm{JB}^{*}$-triple.

Note that by Proposition 2.4(a), $\widetilde{A}$ is a subtriple of $M_{2}(A)$, but we do not know a priori that its triple product is given by (7).

Proof. The proposed triple product, which we denote by $\{\widetilde{a} \widetilde{b} \widetilde{c}\}$, is obviously linear and symmetric in $\widetilde{a}$ and $\widetilde{c}$, and conjugate linear in $\widetilde{b}$. Since, for example,

$$
\{\widetilde{a} \widetilde{b}\{\widetilde{c} \widetilde{d} \widetilde{e}\}\}=\left[\begin{array}{cc}
2\{a b\{c d e\}\} & 2\{a b\{c d e\}\} \\
0 & 0
\end{array}\right],
$$

the main identity (2) is satisfied. 
From $\left\|\left[\begin{array}{cc}a & a \\ 0 & 0\end{array}\right]\right\|=\sqrt{2}\|a\|$ one obtains $\|\{\widetilde{a} \widetilde{a} \widetilde{a}\}\|=\|\widetilde{a}\|^{3},\|\{\widetilde{a} \widetilde{b} \widetilde{c}\}\| \leq$ $\|\widetilde{a}\|\|\widetilde{b}\|\|\widetilde{c}\|$ and hence $\|D(\widetilde{a})\|=\|\widetilde{a}\|^{2}$.

Since $e^{i t D(\widetilde{x})} \widetilde{y}=\left(e^{2 i t D(x)} y \tilde{)},\left\|e^{i t D(\widetilde{x})} \widetilde{y}\right\|=\sqrt{2}\left\|e^{2 i t D(x)} y\right\|=\sqrt{2}\|y\|=\|\widetilde{y}\|\right.$, so $D(\widetilde{x})$ is Hermitian.

Finally, for $\lambda<0$, the inverse of $\lambda-D(\widetilde{x})$ is given by

$$
\widetilde{y} \mapsto\left[\begin{array}{cc}
(\lambda-2 D(x))^{-1} y & (\lambda-2 D(x))^{-1} y \\
0 & 0
\end{array}\right] .
$$

Hence, $S p_{B(\widetilde{A})}(D(\widetilde{x})) \subset[0, \infty)$.

Lemma 3.2. For $a, b, c \in A$, there exist $x, y, z, w \in A$ such that

$$
\left\{\left[\begin{array}{ll}
a & a \\
0 & 0
\end{array}\right]\left[\begin{array}{ll}
0 & b \\
0 & 0
\end{array}\right]\left[\begin{array}{cc}
c & c \\
0 & 0
\end{array}\right]\right\}=\left[\begin{array}{cc}
x & y \\
z & w
\end{array}\right]
$$

and $(x+y) / 2=\{a b c\}$.

Proof. Consider the projection $P$ defined in Proposition 2.4(a). By (5),

$$
\begin{aligned}
& P\left(\left\{\left[\begin{array}{ll}
a & a \\
0 & 0
\end{array}\right]\left[\begin{array}{ll}
0 & b \\
0 & 0
\end{array}\right]\left[\begin{array}{ll}
c & c \\
0 & 0
\end{array}\right]\right\}\right) \\
& =P\left(\left\{\left[\begin{array}{ll}
a & a \\
0 & 0
\end{array}\right]\left[\begin{array}{cc}
b / 2 & b / 2 \\
0 & 0
\end{array}\right]\left[\begin{array}{ll}
c & c \\
0 & 0
\end{array}\right]\right\}\right) .
\end{aligned}
$$

By Remark 3.1 and the uniqueness of the triple product in a $\mathrm{JB}^{*}$-triple,

$$
P\left(\left\{\left[\begin{array}{ll}
a & a \\
0 & 0
\end{array}\right]\left[\begin{array}{ll}
b & b \\
0 & 0
\end{array}\right]\left[\begin{array}{ll}
c & c \\
0 & 0
\end{array}\right]\right\}\right)=2\left[\begin{array}{cc}
\{a b c\} & \{a b c\} \\
0 & 0
\end{array}\right] .
$$

Thus, if

$$
\left\{\left[\begin{array}{ll}
a & a \\
0 & 0
\end{array}\right]\left[\begin{array}{ll}
0 & b \\
0 & 0
\end{array}\right]\left[\begin{array}{ll}
c & c \\
0 & 0
\end{array}\right]\right\}=\left[\begin{array}{cc}
x & y \\
z & w
\end{array}\right]
$$

then

$$
\left[\begin{array}{cc}
\{a b c\} & \{a b c\} \\
0 & 0
\end{array}\right]=\left[\begin{array}{cc}
(x+y) / 2 & (x+y) / 2 \\
0 & 0
\end{array}\right]
$$

It will be shown below in the proof of Lemma 3.8 that $x=y=\{a b c\}$ and that each $z=w=0$.

Lemma 3.3. For each $a, b \in A$,

$$
\left[\begin{array}{ll}
a & 0 \\
0 & 0
\end{array}\right] \perp\left[\begin{array}{ll}
0 & 0 \\
0 & b
\end{array}\right] \text { and }\left[\begin{array}{ll}
0 & 0 \\
a & 0
\end{array}\right] \perp\left[\begin{array}{ll}
0 & b \\
0 & 0
\end{array}\right] \text {. }
$$


Proof. Suppose first that $a=\sum \lambda_{i} u_{i}$ where $\lambda_{i}>0$ and the $u_{i}$ are tripotents in $A$, and similarly for $b=\sum \mu_{j} v_{j}$. Because the image of a bicontractive projection is a subtriple ([20, Proposition 1]), $U_{i}:=\left[\begin{array}{cc}u_{i} & 0 \\ 0 & 0\end{array}\right]$ and $V_{j}:=$ $\left[\begin{array}{cc}0 & 0 \\ 0 & v_{j}\end{array}\right]$ are tripotents, and since they are orthogonal in $B(H \oplus H), \| U_{i} \pm$ $V_{j} \|=1$. Hence $D\left(U_{i}, V_{j}\right)=0$ in (the abstract triple product of) $M_{2}(A)$ and so for all $x, y, z, w \in A$,

$$
\begin{aligned}
& \left\{\left[\begin{array}{ll}
a & 0 \\
0 & 0
\end{array}\right]\left[\begin{array}{ll}
0 & 0 \\
0 & b
\end{array}\right]\left[\begin{array}{cc}
x & y \\
z & w
\end{array}\right]\right\} \\
& =\sum_{i, j} \lambda_{i} \mu_{j}\left\{\left[\begin{array}{cc}
u_{i} & 0 \\
0 & 0
\end{array}\right]\left[\begin{array}{cc}
0 & 0 \\
0 & v_{j}
\end{array}\right]\left[\begin{array}{cc}
x & y \\
z & w
\end{array}\right]\right\}=0 .
\end{aligned}
$$

For the general case, note that, by [16, 3.2.1], there is an operator space structure on the dual of any operator space $A$ such that the canonical inclusion of $A$ into $A^{* *}$ is a complete isometry. Moreover, by [6, Theorem 2.5] the norm structure on $M_{n}\left(A^{* *}\right)$ coincides with that obtained from the identification $M_{n}\left(A^{* *}\right)=M_{n}(A)^{* *}$. Hence, for all $n, M_{n}\left(A^{* *}\right)$ is a $\mathrm{JBW}^{*}$-triple containing $M_{n}(A)$ as subtriple. Since each element of $A$ can be approximated in norm by finite linear combinations of tripotents in $A^{* *}$, the first statement in the lemma follows from the norm continuity of the triple product.

Since interchanging rows is an isometry, hence an isomorphism, the second statement follows.

Lemma 3.4. Let $a, b, c \in A$. Then

$$
\begin{aligned}
& \left\{\left[\begin{array}{ll}
a & 0 \\
0 & 0
\end{array}\right]\left[\begin{array}{ll}
0 & b \\
0 & 0
\end{array}\right]\left[\begin{array}{ll}
c & 0 \\
0 & 0
\end{array}\right]\right\}=0, \\
& \left\{\left[\begin{array}{ll}
a & 0 \\
0 & 0
\end{array}\right]\left[\begin{array}{ll}
0 & 0 \\
b & 0
\end{array}\right]\left[\begin{array}{ll}
c & 0 \\
0 & 0
\end{array}\right]\right\}=0 .
\end{aligned}
$$

Proof. To prove the first statement, let $X$ denote

$$
\left\{\left[\begin{array}{ll}
a & 0 \\
0 & 0
\end{array}\right]\left[\begin{array}{ll}
0 & b \\
0 & 0
\end{array}\right]\left[\begin{array}{ll}
c & 0 \\
0 & 0
\end{array}\right]\right\} \text {. }
$$

By (5),

$$
P_{11}(X)=P_{11}\left(\left\{\left[\begin{array}{ll}
a & 0 \\
0 & 0
\end{array}\right]\left[\begin{array}{ll}
0 & 0 \\
0 & 0
\end{array}\right]\left[\begin{array}{ll}
c & 0 \\
0 & 0
\end{array}\right]\right\}\right)=0
$$

Similarly, $\left(P_{11}+P_{21}\right)(X)=\left(P_{21}+P_{22}\right)(X)=0$, so that $X=\left[\begin{array}{ll}0 & x \\ 0 & 0\end{array}\right]$. 
Let $X^{\prime}=\left[\begin{array}{ll}0 & 0 \\ 0 & x\end{array}\right]$. We claim that for any $Y \in M_{2}(A),\left\{X X^{\prime} Y\right\}=0$. Indeed, with $A=\left[\begin{array}{ll}a & 0 \\ 0 & 0\end{array}\right], B=\left[\begin{array}{ll}0 & b \\ 0 & 0\end{array}\right], C=\left[\begin{array}{ll}c & 0 \\ 0 & 0\end{array}\right]$, we have $A \perp X^{\prime}$, $C \perp X^{\prime}$ and by (4),

$$
\begin{aligned}
\left\{X X^{\prime} Y\right\} & =\left\{\{A B C\} X^{\prime} Y\right\} \\
& =\left\{C\left\{B A X^{\prime}\right\} Y\right\}+\left\{A\left\{X^{\prime} C B\right\} Y\right\}-\left\{\left\{C X^{\prime} A\right\} B Y\right\}=0 .
\end{aligned}
$$

Thus $D\left(X, X^{\prime}\right)=0$, which, by [22, Lemma 1.3(a)], implies that $X$ and $X^{\prime}$ are orthogonal in the Banach space sense: $\left\|X \pm X^{\prime}\right\|=\max \left(\|X\|,\left\|X^{\prime}\right\|\right)$. Since $\left\|X+X^{\prime}\right\|=\left\|\left[\begin{array}{ll}0 & x \\ 0 & x\end{array}\right]\right\|=\sqrt{2}\|x\|$, it follows that $x=0$. The second assertion is proved similarly, using $X=\left[\begin{array}{ll}0 & 0 \\ x & 0\end{array}\right], X^{\prime}=\left[\begin{array}{ll}0 & 0 \\ 0 & x\end{array}\right]$.

By interchanging rows and columns, it follows that the following triple products all vanish (the last three by orthogonality):

$$
\begin{array}{ll}
\text { (9) }\left\{\left[\begin{array}{ll}
0 & 0 \\
0 & a
\end{array}\right]\left[\begin{array}{ll}
0 & 0 \\
b & 0
\end{array}\right]\left[\begin{array}{ll}
0 & 0 \\
0 & c
\end{array}\right]\right\}, & \left\{\left[\begin{array}{ll}
0 & a \\
0 & 0
\end{array}\right]\left[\begin{array}{ll}
b & 0 \\
0 & 0
\end{array}\right]\left[\begin{array}{ll}
0 & c \\
0 & 0
\end{array}\right]\right\}, \\
(10) \quad\left\{\left[\begin{array}{ll}
0 & 0 \\
a & 0
\end{array}\right]\left[\begin{array}{ll}
0 & 0 \\
0 & b
\end{array}\right]\left[\begin{array}{ll}
0 & 0 \\
c & 0
\end{array}\right]\right\}, & \left\{\left[\begin{array}{ll}
0 & 0 \\
a & 0
\end{array}\right]\left[\begin{array}{ll}
0 & b \\
0 & 0
\end{array}\right]\left[\begin{array}{ll}
0 & 0 \\
0 & c
\end{array}\right]\right\}, \\
\text { (11) }\left\{\left[\begin{array}{ll}
0 & a \\
0 & 0
\end{array}\right]\left[\begin{array}{ll}
0 & 0 \\
b & 0
\end{array}\right]\left[\begin{array}{ll}
c & 0 \\
0 & 0
\end{array}\right]\right\}, & \left\{\left[\begin{array}{ll}
0 & 0 \\
0 & a
\end{array}\right]\left[\begin{array}{ll}
b & 0 \\
0 & 0
\end{array}\right]\left[\begin{array}{ll}
0 & 0 \\
c & 0
\end{array}\right]\right\} .
\end{array}
$$

For use in Lemma 5.2, we adjoin

$$
\left\{\left[\begin{array}{ll}
0 & 0 \\
a & 0
\end{array}\right]\left[\begin{array}{ll}
b & 0 \\
0 & 0
\end{array}\right]\left[\begin{array}{ll}
0 & 0 \\
c & 0
\end{array}\right]\right\}=\left\{\left[\begin{array}{ll}
0 & a \\
0 & 0
\end{array}\right]\left[\begin{array}{ll}
0 & 0 \\
0 & b
\end{array}\right]\left[\begin{array}{ll}
0 & c \\
0 & 0
\end{array}\right]\right\}=0,
$$

and

$$
\left\{\left[\begin{array}{ll}
0 & 0 \\
0 & a
\end{array}\right]\left[\begin{array}{ll}
0 & b \\
0 & 0
\end{array}\right]\left[\begin{array}{ll}
0 & 0 \\
0 & c
\end{array}\right]\right\}=0 \text {. }
$$

Lemma 3.5. For $a, b, c \in A$, there exists $z \in A$ such that

$$
\left\{\left[\begin{array}{ll}
a & 0 \\
0 & 0
\end{array}\right]\left[\begin{array}{ll}
0 & b \\
0 & 0
\end{array}\right]\left[\begin{array}{ll}
0 & c \\
0 & 0
\end{array}\right]\right\}=\left[\begin{array}{ll}
z & 0 \\
0 & 0
\end{array}\right] \text {. }
$$

Proof. Let $X$ denote $\left\{\left[\begin{array}{ll}a & 0 \\ 0 & 0\end{array}\right]\left[\begin{array}{ll}0 & b \\ 0 & 0\end{array}\right]\left[\begin{array}{ll}0 & c \\ 0 & 0\end{array}\right]\right\}$. By $(5),\left(P_{12}+P_{22}\right)(X)$ $=0$ and $\left(P_{12}+P_{21}\right)(X)=0$. 
Lemma 3.6. For $a, b, c \in A$,

$$
\left\{\left[\begin{array}{ll}
0 & a \\
0 & 0
\end{array}\right]\left[\begin{array}{ll}
0 & b \\
0 & 0
\end{array}\right]\left[\begin{array}{ll}
0 & c \\
0 & 0
\end{array}\right]\right\}=\left[\begin{array}{cc}
0 & \{a b c\} \\
0 & 0
\end{array}\right] .
$$

Proof. Since $P_{11}+P_{12}$ and $P_{12}+P_{22}$ are bicontractive, the intersection of their ranges is a subtriple. Since $A$ is a $\mathrm{JB}^{*}$-triple under the product induced by $P_{12}$, and triple products are unique, the result follows.

As noted in the proof of Lemma 3.3, interchanging rows or columns is an isometry, hence an isomorphism. Therefore we also have, for example,

$$
\left\{\left[\begin{array}{ll}
a & 0 \\
0 & 0
\end{array}\right]\left[\begin{array}{ll}
b & 0 \\
0 & 0
\end{array}\right]\left[\begin{array}{ll}
c & 0 \\
0 & 0
\end{array}\right]\right\}=\left[\begin{array}{cc}
\{a b c\} & 0 \\
0 & 0
\end{array}\right]
$$

and so forth.

In Proposition 2.4(b) we have defined projections $P_{i j}: M_{n}(A) \rightarrow M_{n}(A)$ as follows: If $X=\left[x_{k l}\right] \in M_{n}(A)$, then $P_{i j}(X)$ is the element of $M_{n}(A)$ with $x_{i j}$ in the $(i, j)$ entry and zeros elsewhere. In what follows, we shall use the maps $p_{i j}: M_{n}(A) \rightarrow A$ defined by $p_{i j}(X)=x_{i j}$ for $X=\left[x_{k l}\right] \in M_{n}(A)$.

Definition 3.7. Define a ternary product $[a, b, c]$ or $[a b c]$ on $A$ by

$$
[a, b, c]=2 p_{11}\left(\left\{\left[\begin{array}{ll}
0 & a \\
0 & 0
\end{array}\right]\left[\begin{array}{ll}
0 & b \\
0 & 0
\end{array}\right]\left[\begin{array}{ll}
c & 0 \\
0 & 0
\end{array}\right]\right\}\right) .
$$

Similarly, define two more ternary products $(a b c)$ and $\langle a b c\rangle$ as follows:

$$
(a b c)=2 p_{11}\left(\left\{\left[\begin{array}{ll}
a & 0 \\
0 & 0
\end{array}\right]\left[\begin{array}{ll}
0 & 0 \\
b & 0
\end{array}\right]\left[\begin{array}{ll}
0 & 0 \\
c & 0
\end{array}\right]\right\}\right)
$$

and

$$
\langle a b c\rangle=2 p_{11}\left(\left\{\left[\begin{array}{ll}
0 & 0 \\
c & 0
\end{array}\right]\left[\begin{array}{ll}
0 & 0 \\
0 & b
\end{array}\right]\left[\begin{array}{ll}
0 & a \\
0 & 0
\end{array}\right]\right\}\right) .
$$

We treat first the ternary product $[a, b, c]$. Note that, by Lemma 3.5,

$$
\frac{1}{2}\left[\begin{array}{cc}
{[a, b, c]} & 0 \\
0 & 0
\end{array}\right]=\left\{\left[\begin{array}{ll}
0 & a \\
0 & 0
\end{array}\right]\left[\begin{array}{ll}
0 & b \\
0 & 0
\end{array}\right]\left[\begin{array}{ll}
c & 0 \\
0 & 0
\end{array}\right]\right\},
$$

and that by interchanging suitable rows and columns,

$$
\begin{aligned}
{[a, b, c] } & =2 p_{21}\left(\left\{\left[\begin{array}{ll}
0 & 0 \\
0 & a
\end{array}\right]\left[\begin{array}{ll}
0 & 0 \\
0 & b
\end{array}\right]\left[\begin{array}{ll}
0 & 0 \\
c & 0
\end{array}\right]\right\}\right) \\
& =2 p_{12}\left(\left\{\left[\begin{array}{ll}
a & 0 \\
0 & 0
\end{array}\right]\left[\begin{array}{ll}
b & 0 \\
0 & 0
\end{array}\right]\left[\begin{array}{ll}
0 & c \\
0 & 0
\end{array}\right]\right\}\right) \\
& =2 p_{22}\left(\left\{\left[\begin{array}{ll}
0 & 0 \\
a & 0
\end{array}\right]\left[\begin{array}{ll}
0 & 0 \\
b & 0
\end{array}\right]\left[\begin{array}{ll}
0 & 0 \\
0 & c
\end{array}\right]\right\}\right) .
\end{aligned}
$$


Lemma 3.8. For $a, b, c \in A$,

$$
[a, b, c]+[c, b, a]=2\{a b c\},
$$

and hence

$$
\|[a, a, a]\|=\|a\|^{3} .
$$

Proof. Given $a, b, c \in A$, it follows from Lemma 3.2, Lemmas 3.4-3.6, Definition 3.7 and (6) that there are elements $x, y, z, w \in A$ such that $x+y=$ $2\{a b c\}$ and

$$
\left[\begin{array}{cc}
x & y \\
z & w
\end{array}\right]=\left[\begin{array}{ll}
0 & 0 \\
0 & 0
\end{array}\right]+\left[\begin{array}{cc}
{[a b c] / 2} & 0 \\
0 & 0
\end{array}\right]+\left[\begin{array}{cc}
0 & \{a b c\} \\
0 & 0
\end{array}\right]+\left[\begin{array}{cc}
{[c b a] / 2} & 0 \\
0 & 0
\end{array}\right] .
$$

Hence $[a b c] / 2+[c b a] / 2=x=y=\{a b c\}$ (and $z=w=0$ ).

We shall see later in Proposition 4.5 that in fact $[a b c]=(a b c)=\langle a b c\rangle$. First we shall show the analog of Lemma 3.8 for each of the ternary products $(a b c)$ and $\langle a b c\rangle$. We note that, as above,

$$
\left[\begin{array}{cc}
(a b c) / 2 & 0 \\
0 & 0
\end{array}\right]=\left\{\left[\begin{array}{ll}
a & 0 \\
0 & 0
\end{array}\right]\left[\begin{array}{ll}
0 & 0 \\
b & 0
\end{array}\right]\left[\begin{array}{ll}
0 & 0 \\
c & 0
\end{array}\right]\right\}
$$

and

$$
\left[\begin{array}{cc}
\langle a b c\rangle / 2 & 0 \\
0 & 0
\end{array}\right]=\left\{\left[\begin{array}{ll}
0 & 0 \\
c & 0
\end{array}\right]\left[\begin{array}{ll}
0 & 0 \\
0 & b
\end{array}\right]\left[\begin{array}{ll}
0 & a \\
0 & 0
\end{array}\right]\right\}
$$

Moreover, by interchanging rows and/or columns,

$$
\begin{aligned}
(a b c) & =2 p_{22}\left(\left\{\left[\begin{array}{ll}
0 & 0 \\
0 & a
\end{array}\right]\left[\begin{array}{ll}
0 & b \\
0 & 0
\end{array}\right]\left[\begin{array}{ll}
0 & c \\
0 & 0
\end{array}\right]\right\}\right) \\
& =2 p_{12}\left(\left\{\left[\begin{array}{ll}
0 & a \\
0 & 0
\end{array}\right]\left[\begin{array}{ll}
0 & 0 \\
0 & b
\end{array}\right]\left[\begin{array}{ll}
0 & 0 \\
0 & c
\end{array}\right]\right\}\right) \\
& =2 p_{21}\left(\left\{\left[\begin{array}{ll}
0 & 0 \\
a & 0
\end{array}\right]\left[\begin{array}{ll}
b & 0 \\
0 & 0
\end{array}\right]\left[\begin{array}{ll}
c & 0 \\
0 & 0
\end{array}\right]\right\}\right)
\end{aligned}
$$

and

$$
\begin{aligned}
\langle a b c\rangle & =2 p_{22}\left(\left\{\left[\begin{array}{ll}
0 & c \\
0 & 0
\end{array}\right]\left[\begin{array}{ll}
b & 0 \\
0 & 0
\end{array}\right]\left[\begin{array}{ll}
0 & 0 \\
a & 0
\end{array}\right]\right\}\right) \\
& =2 p_{12}\left(\left\{\left[\begin{array}{ll}
0 & 0 \\
0 & c
\end{array}\right]\left[\begin{array}{ll}
0 & 0 \\
b & 0
\end{array}\right]\left[\begin{array}{ll}
a & 0 \\
0 & 0
\end{array}\right]\right\}\right) \\
& =2 p_{21}\left(\left\{\left[\begin{array}{ll}
c & 0 \\
0 & 0
\end{array}\right]\left[\begin{array}{ll}
0 & b \\
0 & 0
\end{array}\right]\left[\begin{array}{ll}
0 & 0 \\
0 & a
\end{array}\right]\right\}\right) .
\end{aligned}
$$

Proposition 3.9. If $A$ is an operator space such that $M_{2}(A)_{0}$ is a bounded symmetric domain (and consequently $M_{2}(A)$ and $A$ are $\mathrm{JB}^{*}$-triples), then $\langle a b c\rangle+\langle c b a\rangle=2\{a b c\}_{A}$ and $(a b c)+(c b a)=2\{a b c\}_{A}$. 
Proof. The proof for $(\cdot, \cdot, \cdot)$ is similar to the proof for $[\cdot, \cdot, \cdot]$, using instead the identity

$$
\begin{aligned}
\left\{\left[\begin{array}{ll}
a & 0 \\
a & 0
\end{array}\right]\left[\begin{array}{ll}
0 & 0 \\
b & 0
\end{array}\right]\left[\begin{array}{ll}
c & 0 \\
c & 0
\end{array}\right]\right\}= & \left\{\left[\begin{array}{ll}
0 & 0 \\
c & 0
\end{array}\right]\left[\begin{array}{ll}
0 & 0 \\
b & 0
\end{array}\right]\left[\begin{array}{ll}
0 & 0 \\
a & 0
\end{array}\right]\right\} \\
& +\left\{\left[\begin{array}{ll}
c & 0 \\
0 & 0
\end{array}\right]\left[\begin{array}{ll}
0 & 0 \\
b & 0
\end{array}\right]\left[\begin{array}{ll}
0 & 0 \\
a & 0
\end{array}\right]\right\} \\
& +\left\{\left[\begin{array}{ll}
a & 0 \\
0 & 0
\end{array}\right]\left[\begin{array}{ll}
0 & 0 \\
b & 0
\end{array}\right]\left[\begin{array}{ll}
c & 0 \\
0 & 0
\end{array}\right]\right\} \\
& +\left\{\left[\begin{array}{ll}
a & 0 \\
0 & 0
\end{array}\right]\left[\begin{array}{ll}
0 & 0 \\
b & 0
\end{array}\right]\left[\begin{array}{ll}
0 & 0 \\
c & 0
\end{array}\right]\right\}
\end{aligned}
$$

and the projection

$$
P\left(\left[\begin{array}{ll}
a & b \\
c & d
\end{array}\right]\right)=\frac{1}{2}\left[\begin{array}{ll}
a+c & 0 \\
a+c & 0
\end{array}\right] .
$$

To prove the statement for $\langle\cdot, \cdot, \cdot\rangle$ consider (cf. Remark 3.1) the space

$$
\widetilde{A}=\left\{\widetilde{a}=\left[\begin{array}{ll}
a & a \\
a & a
\end{array}\right]: a \in A\right\},
$$

which is a subtriple of $M_{2}(A)$ since it is the range of a product $Q P$ of the bicontractive projections $Q, P$ of Proposition 2.4(c). It follows as in the proof of Remark 3.1 that $\widetilde{A}$ is a JB*-triple under the triple product $\{\cdots\}^{\prime}$ defined by $\{\widetilde{a} \widetilde{b} \widetilde{c}\}^{\prime}=4(\{a b c\})^{\widetilde{2}}$. To see this, let $D^{\prime}(\widetilde{x}) \widetilde{a}=\{\widetilde{x} \widetilde{x} \widetilde{a}\}^{\prime}$ and note that $\|\widetilde{x}\|=2\|x\|, D^{\prime}(\widetilde{x}) \widetilde{a}=4(D(x) a) \widetilde{r}, e^{i t D^{\prime}(\widetilde{x})} \widetilde{y}=\left(e^{4 i t D(x)} y\right)^{\widetilde{y}}$ and that $(\lambda-D(\widetilde{x}))^{-1} \widetilde{y}=\left((\lambda-D(x))^{-1} y\right)^{-}$. By the uniqueness of the triple product on $M_{2}(A),\{\widetilde{a} \widetilde{b} \widetilde{c}\}=\{\widetilde{a} \widetilde{b} \widetilde{c}\}^{\prime}$. Hence, by expanding $\{\widetilde{x} \widetilde{y} \widetilde{z}\}=$ $\left\{\left[\begin{array}{ll}x & x \\ x & x\end{array}\right]\left[\begin{array}{ll}y & y \\ y & y\end{array}\right]\left[\begin{array}{ll}z & z \\ z & z\end{array}\right]\right\}$ into computable terms,

$$
\begin{aligned}
& 4\{x y z \tilde{z} \\
& =\{\widetilde{x} \widetilde{y}\} \\
& =(\{x y z\}+(x y z) / 2+(z y x) / 2+[x y z] / 2+[z y x] / 2+\langle x y z\rangle / 2+\langle z y x\rangle / 2) \\
& =(3\{x y z\}+\langle x y z\rangle / 2+\langle z y x\rangle / 2) \tilde{.} .
\end{aligned}
$$

This proves the statement for $\langle\cdot, \cdot, \cdot\rangle$.

\section{Equality of the ternary products.}

In this section, we continue to assume that $A \subset B(H)$ is an operator space such that the open unit ball $M_{2}(A)_{0}$ is a bounded symmetric domain. We shall prove the equality of the three ternary products defined in Section 3. 
Even though they agree, all three products are needed in the proof of the crucial Proposition 5.1.

In the following we shall let $\mathbf{a} \in M_{2}(A)$ denote $\left[\begin{array}{ll}a & 0 \\ 0 & a\end{array}\right]$ and $\overline{\mathbf{a}} \in M_{2}(A)$ denote $\left[\begin{array}{ll}0 & a \\ a & 0\end{array}\right]$. By Lemmas 3.3 and 3.6, the ranges of $P_{12}+P_{21}$ and $P_{11}+P_{22}$ are invariant under the continuous functional calculus in a JB*triple. In particular, for any $\lambda>0$,

$$
\mathbf{a}^{\lambda}=\left[\begin{array}{cc}
a^{\lambda} & 0 \\
0 & a^{\lambda}
\end{array}\right] \text { and }(\overline{\mathbf{a}})^{\lambda}=\left[\begin{array}{cc}
0 & a^{\lambda} \\
a^{\lambda} & 0
\end{array}\right]
$$

Here, $\mathbf{a}^{\lambda}$ is defined by the triple functional calculus in the JB*-triple $M_{2}(A)$ and $a^{\lambda}$ is defined by the triple functional calculus in the $\mathrm{JB}^{*}$-triple $A$.

Lemma 4.1. Let $\lambda, \mu, \nu$ be positive numbers and let $a \in A$. Then

$$
\mathbf{a}^{\lambda+\mu+\nu}=\left\{\mathbf{a}^{\lambda} \mathbf{a}^{\mu} \mathbf{a}^{\nu}\right\}=\left\{\overline{\mathbf{a}}^{\lambda} \mathbf{a}^{\mu} \overline{\mathbf{a}}^{\nu}\right\}=\left\{\overline{\mathbf{a}}^{\lambda} \overline{\mathbf{a}}^{\mu} \mathbf{a}^{\nu}\right\}
$$

and

$$
\overline{\mathbf{a}}^{\lambda+\mu+\nu}=\left\{\overline{\mathbf{a}}^{\lambda} \overline{\mathbf{a}}^{\mu} \overline{\mathbf{a}}^{\nu}\right\}=\left\{\mathbf{a}^{\lambda} \overline{\mathbf{a}}^{\mu} \mathbf{a}^{\nu}\right\}=\left\{\overline{\mathbf{a}}^{\lambda} \mathbf{a}^{\mu} \mathbf{a}^{\nu}\right\}
$$

Proof. $\mathbf{a}^{\lambda+\mu+\nu}=\left\{\mathbf{a}^{\lambda} \mathbf{a}^{\mu} \mathbf{a}^{\nu}\right\}$ is immediate from the functional calculus. The proofs of the other statements are all proved in the same way, for example,

$$
\begin{aligned}
\left\{\overline{\mathbf{a}}^{\lambda} \mathbf{a}^{\mu} \overline{\mathbf{a}}^{\nu}\right\}= & \left\{\left[\begin{array}{cc}
0 & a^{\lambda} \\
a^{\lambda} & 0
\end{array}\right]\left[\begin{array}{cc}
a^{\mu} & 0 \\
0 & a^{\mu}
\end{array}\right]\left[\begin{array}{cc}
0 & a^{\nu} \\
a^{\nu} & 0
\end{array}\right]\right\} \\
= & \left\{\left[\begin{array}{cc}
0 & a^{\lambda} \\
0 & 0
\end{array}\right]\left[\begin{array}{cc}
a^{\mu} & 0 \\
0 & 0
\end{array}\right]\left[\begin{array}{cc}
0 & a^{\nu} \\
a^{\nu} & 0
\end{array}\right]\right\} \\
& +\left\{\left[\begin{array}{cc}
0 & a^{\lambda} \\
0 & 0
\end{array}\right]\left[\begin{array}{cc}
0 & 0 \\
0 & a^{\mu}
\end{array}\right]\left[\begin{array}{cc}
0 & a^{\nu} \\
a^{\nu} & 0
\end{array}\right]\right\} \\
& +\left\{\left[\begin{array}{cc}
0 & 0 \\
a^{\lambda} & 0
\end{array}\right]\left[\begin{array}{cc}
a^{\mu} & 0 \\
0 & 0
\end{array}\right]\left[\begin{array}{cc}
0 & a^{\nu} \\
a^{\nu} & 0
\end{array}\right]\right\} \\
& +\left\{\left[\begin{array}{cc}
0 & 0 \\
a^{\lambda} & 0
\end{array}\right]\left[\begin{array}{cc}
0 & 0 \\
0 & a^{\mu}
\end{array}\right]\left[\begin{array}{cc}
0 & a^{\nu} \\
a^{\nu} & 0
\end{array}\right]\right\}
\end{aligned}
$$


which further expands, using (8)-(11) into

$$
\begin{aligned}
& \left\{\left[\begin{array}{cc}
0 & a^{\lambda} \\
0 & 0
\end{array}\right]\left[\begin{array}{cc}
a^{\mu} & 0 \\
0 & 0
\end{array}\right]\left[\begin{array}{cc}
0 & 0 \\
a^{\nu} & 0
\end{array}\right]\right\} \\
& +\left\{\left[\begin{array}{cc}
0 & a^{\lambda} \\
0 & 0
\end{array}\right]\left[\begin{array}{cc}
0 & 0 \\
0 & a^{\mu}
\end{array}\right]\left[\begin{array}{cc}
0 & 0 \\
a^{\nu} & 0
\end{array}\right]\right\} \\
& +\left\{\left[\begin{array}{cc}
0 & 0 \\
a^{\lambda} & 0
\end{array}\right]\left[\begin{array}{cc}
a^{\mu} & 0 \\
0 & 0
\end{array}\right]\left[\begin{array}{cc}
0 & a^{\nu} \\
0 & 0
\end{array}\right]\right\} \\
& +\left\{\left[\begin{array}{cc}
0 & 0 \\
a^{\lambda} & 0
\end{array}\right]\left[\begin{array}{cc}
0 & 0 \\
0 & a^{\mu}
\end{array}\right]\left[\begin{array}{cc}
0 & a^{\nu} \\
0 & 0
\end{array}\right]\right\} \\
& =\left[\begin{array}{ccc}
0 & 0 \\
0 & \left\langle a^{\nu} a^{\mu} a^{\lambda}\right\rangle / 2
\end{array}\right]+\left[\begin{array}{cc}
\left\langle a^{\lambda} a^{\mu} a^{\nu}\right\rangle / 2 & 0 \\
0 & 0
\end{array}\right] \\
& \quad+\left[\begin{array}{cc}
0 & 0 \\
0 & \left\langle a^{\lambda} a^{\mu} a^{\nu}\right\rangle / 2
\end{array}\right]+\left[\begin{array}{cc}
\left\langle a^{\nu} a^{\mu} a^{\lambda}\right\rangle / 2 & 0 \\
0 & 0
\end{array}\right]=\mathbf{a}^{\lambda+\mu+\nu} .
\end{aligned}
$$

Lemma 4.2. $D(\mathbf{a}, \mathbf{a})=D(\overline{\mathbf{a}}, \overline{\mathbf{a}})$.

Proof. We shall use (3) with $z=\{x x y\}$, which states that

$$
D(\{x y x\},\{x x y\})=2 D(x,\{y x\{x x y\}\})-D(\{x\{x x y\} x\}, y) .
$$

We have, by (17) and Lemma 4.1,

$$
\begin{aligned}
D(\mathbf{a}, \mathbf{a})= & D\left(\left\{\overline{\mathbf{a}}^{1 / 3}, \mathbf{a}^{1 / 3}, \overline{\mathbf{a}}^{1 / 3}\right\},\left\{\overline{\mathbf{a}}^{1 / 3}, \overline{\mathbf{a}}^{1 / 3}, \mathbf{a}^{1 / 3}\right\}\right) \\
= & 2 D\left(\overline{\mathbf{a}}^{1 / 3},\left\{\mathbf{a}^{1 / 3}, \overline{\mathbf{a}}^{1 / 3},\left\{\overline{\mathbf{a}}^{1 / 3}, \overline{\mathbf{a}}^{1 / 3}, \mathbf{a}^{1 / 3}\right\}\right\}\right) \\
& -D\left(\left\{\overline{\mathbf{a}}^{1 / 3},\left\{\overline{\mathbf{a}}^{1 / 3}, \overline{\mathbf{a}}^{1 / 3}, \mathbf{a}^{1 / 3}\right\}, \overline{\mathbf{a}}^{1 / 3}\right\}, \mathbf{a}^{1 / 3}\right) \\
= & 2 D\left(\overline{\mathbf{a}}^{1 / 3},\left\{\mathbf{a}^{1 / 3}, \overline{\mathbf{a}}^{1 / 3}, \mathbf{a}\right\}\right)-D\left(\left\{\overline{\mathbf{a}}^{1 / 3}, \mathbf{a}, \overline{\mathbf{a}}^{1 / 3}\right\}, \mathbf{a}^{1 / 3}\right) \\
= & 2 D\left(\overline{\mathbf{a}}^{1 / 3}, \overline{\mathbf{a}}^{5 / 3}\right)-D\left(\mathbf{a}^{5 / 3}, \mathbf{a}^{1 / 3}\right) \\
= & 2 D(\overline{\mathbf{a}}, \overline{\mathbf{a}})-D(\mathbf{a}, \mathbf{a}),
\end{aligned}
$$

which proves the lemma.

Lemma 4.3. $D(\mathbf{a}, \overline{\mathbf{a}})=D(\overline{\mathbf{a}}, \mathbf{a})$. 
Proof. By Lemma 4.1 and two applications of (1),

$$
\begin{aligned}
D(\mathbf{a}, \overline{\mathbf{a}})= & D\left(\left\{\mathbf{a}^{1 / 4}, \overline{\mathbf{a}}^{1 / 4}, \overline{\mathbf{a}}^{1 / 2}\right\}, \overline{\mathbf{a}}\right) \\
= & D\left(\overline{\mathbf{a}}^{1 / 2},\left\{\overline{\mathbf{a}} \mathbf{a}^{1 / 4} \overline{\mathbf{a}}^{1 / 4}\right\}\right)+\left[D\left(\mathbf{a}^{1 / 4}, \overline{\mathbf{a}}^{1 / 4}\right), D\left(\overline{\mathbf{a}}^{1 / 2}, \overline{\mathbf{a}}\right)\right] \\
= & D\left(\overline{\mathbf{a}}^{1 / 2}, \mathbf{a}^{3 / 2}\right)+\left[D\left(\mathbf{a}^{1 / 4}, \overline{\mathbf{a}}^{1 / 4}\right), D\left(\mathbf{a}^{1 / 2}, \mathbf{a}\right)\right] \\
& \left(\text { by Lemma } 4.2 \text { since } D\left(\overline{\mathbf{a}}^{1 / 2}, \overline{\mathbf{a}}\right)=D\left(\overline{\mathbf{a}}^{3 / 4}, \overline{\mathbf{a}}^{3 / 4}\right)\right) \\
= & D\left(\overline{\mathbf{a}}^{1 / 2}, \mathbf{a}^{3 / 2}\right)+D\left(\left\{\mathbf{a}^{1 / 4} \overline{\mathbf{a}}^{1 / 4} \mathbf{a}^{1 / 2}\right\}, \mathbf{a}\right)-D\left(\mathbf{a}^{1 / 2},\left\{\mathbf{a a}^{1 / 4} \overline{\mathbf{a}}^{1 / 4}\right\}\right) \\
= & D\left(\overline{\mathbf{a}}^{1 / 2}, \mathbf{a}^{3 / 2}\right)+D(\overline{\mathbf{a}}, \mathbf{a})-D\left(\mathbf{a}^{1 / 2}, \overline{\mathbf{a}}^{3 / 2}\right) .
\end{aligned}
$$

Hence $D(\mathbf{a}, \overline{\mathbf{a}})-D(\overline{\mathbf{a}}, \mathbf{a})=D\left(\overline{\mathbf{a}}^{1 / 2}, \mathbf{a}^{3 / 2}\right)-D\left(\mathbf{a}^{1 / 2}, \overline{\mathbf{a}}^{3 / 2}\right)$.

It remains to show that $D\left(\overline{\mathbf{a}}, \mathbf{a}^{3}\right)-D\left(\mathbf{a}, \overline{\mathbf{a}}^{3}\right)=0$ for every $a \in A$. Now by (3) and Lemma 4.1,

$$
\begin{aligned}
D\left(\overline{\mathbf{a}}, \mathbf{a}^{3}\right) & =D(\overline{\mathbf{a}},\{\mathbf{a}, \overline{\mathbf{a}}, \overline{\mathbf{a}}\}) \\
& =D(\{\overline{\mathbf{a}} \mathbf{a} \overline{\mathbf{a}}\}, \overline{\mathbf{a}}) / 2+D\left(\overline{\mathbf{a}}^{3}, \mathbf{a}\right) / 2 \\
& =D\left(\mathbf{a}^{3}, \overline{\mathbf{a}}\right) / 2+D\left(\overline{\mathbf{a}}^{3}, \mathbf{a}\right) / 2 \\
& \left.=D\left(\mathbf{a}, \overline{\mathbf{a}}^{3}\right) \text { (by interchanging } \mathbf{a} \text { and } \overline{\mathbf{a}}\right) .
\end{aligned}
$$

This proves the lemma.

By linearization from the preceding two lemmas we obtain:

Lemma 4.4. $D(\mathbf{a}, \mathbf{b})=D(\overline{\mathbf{a}}, \overline{\mathbf{b}}) ; D(\overline{\mathbf{a}}, \mathbf{b})=D(\mathbf{a}, \overline{\mathbf{b}})$.

Proof. From $D(\mathbf{a}+\mathbf{b}, \overline{\mathbf{a}}+\overline{\mathbf{b}})=D(\overline{\mathbf{a}}+\overline{\mathbf{b}}, \mathbf{a}+\mathbf{b})$ follows $D(\mathbf{b}, \overline{\mathbf{a}})+D(\mathbf{a}, \overline{\mathbf{b}})=$ $D(\overline{\mathbf{a}}, \mathbf{b})+D(\overline{\mathbf{b}}, \mathbf{a})$. Now replace $a$ by $i a$ and add to obtain $D(\mathbf{a}, \overline{\mathbf{b}})=D(\overline{\mathbf{a}}, \mathbf{b})$. The second statement follows similarly from $D(\mathbf{a}+\mathbf{b}, \mathbf{a}+\mathbf{b})=D(\overline{\mathbf{a}}+\overline{\mathbf{b}}, \overline{\mathbf{a}}+$ $\overline{\mathbf{b}})$.

Proposition 4.5. If $A$ is an operator space such that $M_{2}(A)_{0}$ is a bounded symmetric domain, then $[a b c]=(a b c)=\langle a b c\rangle$.

Proof. By expanding as in the second part of the proof of Lemma 4.3,

$$
\begin{aligned}
D(\mathbf{a}, \mathbf{b})\left[\begin{array}{ll}
x & 0 \\
0 & 0
\end{array}\right] & =\left\{\left[\begin{array}{ll}
a & 0 \\
0 & a
\end{array}\right]\left[\begin{array}{ll}
b & 0 \\
0 & b
\end{array}\right]\left[\begin{array}{ll}
x & 0 \\
0 & 0
\end{array}\right]\right\} \\
& =\left\{\left[\begin{array}{ll}
a & 0 \\
0 & 0
\end{array}\right]\left[\begin{array}{ll}
b & 0 \\
0 & 0
\end{array}\right]\left[\begin{array}{ll}
x & 0 \\
0 & 0
\end{array}\right]\right\} \\
& =\left[\begin{array}{cc}
\{a b x\} & 0 \\
0 & 0
\end{array}\right]
\end{aligned}
$$


and

$$
\begin{aligned}
D(\overline{\mathbf{a}}, \overline{\mathbf{b}})\left[\begin{array}{ll}
x & 0 \\
0 & 0
\end{array}\right]= & \left\{\left[\begin{array}{ll}
0 & a \\
a & 0
\end{array}\right]\left[\begin{array}{ll}
0 & b \\
b & 0
\end{array}\right]\left[\begin{array}{ll}
x & 0 \\
0 & 0
\end{array}\right]\right\} \\
= & \left\{\left[\begin{array}{ll}
0 & a \\
0 & 0
\end{array}\right]\left[\begin{array}{ll}
0 & b \\
0 & 0
\end{array}\right]\left[\begin{array}{ll}
x & 0 \\
0 & 0
\end{array}\right]\right\} \\
& +\left\{\left[\begin{array}{ll}
0 & 0 \\
a & 0
\end{array}\right]\left[\begin{array}{ll}
0 & 0 \\
b & 0
\end{array}\right]\left[\begin{array}{ll}
x & 0 \\
0 & 0
\end{array}\right]\right\} \\
= & {\left[\begin{array}{cc}
{[a b x] / 2+(x b a) / 2} & 0 \\
0 & 0
\end{array}\right], }
\end{aligned}
$$

so that $[x b a]=(x b a)$.

Similarly,

$$
\begin{aligned}
& {\left[\begin{array}{cc}
0 & \langle x b a\rangle / 2 \\
\langle a b x\rangle / 2 & 0
\end{array}\right]=\left\{\left[\begin{array}{ll}
a & 0 \\
0 & a
\end{array}\right]\left[\begin{array}{ll}
0 & b \\
b & 0
\end{array}\right]\left[\begin{array}{ll}
x & 0 \\
0 & 0
\end{array}\right]\right\}} \\
& =D(\mathbf{a}, \overline{\mathbf{b}})\left[\begin{array}{ll}
x & 0 \\
0 & 0
\end{array}\right] \\
& =D(\overline{\mathbf{a}}, \mathbf{b})\left[\begin{array}{ll}
x & 0 \\
0 & 0
\end{array}\right] \\
& =\left\{\left[\begin{array}{ll}
0 & a \\
a & 0
\end{array}\right]\left[\begin{array}{ll}
b & 0 \\
0 & b
\end{array}\right]\left[\begin{array}{ll}
x & 0 \\
0 & 0
\end{array}\right]\right\} \\
& =\left[\begin{array}{cc}
0 & {[x b a] / 2} \\
(a b x) / 2 & 0
\end{array}\right] \text {, }
\end{aligned}
$$

so that $\langle x b a\rangle=[x b a]$.

\section{Main result.}

Proposition 5.1. Let $X$ be an operator space such that $M_{2}(X)_{0}$ is a bounded symmetric domain. Then $(X,[\cdots],\|\cdot\|)$ is a $\mathrm{C}^{*}$-ternary ring in the sense of Zettl [39] (see Definition 2.3) and its $\mathrm{JB}^{*}$-triple product (see the beginning of Section 3) satisfies $\{a b c\}=([a b c]+[c b a]) / 2$.

Proof. It was already shown in Lemma 3.8 that $\{a b c\}=([a b c]+[c b a]) / 2$ and that $\|[a a a]\|=\|a\|^{3}$ and it is clear that $\|[a b c]\| \leq\|a\|\|b\|\|c\|$. It remains to show associativity. To prove this we will use Lemma 3.3 and Proposition 4.5. For $a, b, c, d, e \in X$, let

$$
A=\left[\begin{array}{ll}
0 & a \\
0 & 0
\end{array}\right], B=\left[\begin{array}{ll}
0 & b \\
0 & 0
\end{array}\right], C=\left[\begin{array}{cc}
c & 0 \\
0 & 0
\end{array}\right], D=\left[\begin{array}{ll}
0 & 0 \\
d & 0
\end{array}\right], E=\left[\begin{array}{ll}
0 & 0 \\
e & 0
\end{array}\right] .
$$


Then

$$
\begin{aligned}
{[} & {[a b c] d e] } \\
= & ([a b c] d e)=2 p_{11}\left(\left\{\left[\begin{array}{cc}
{[a b c]} & 0 \\
0 & 0
\end{array}\right],\left[\begin{array}{ll}
0 & 0 \\
d & 0
\end{array}\right],\left[\begin{array}{ll}
0 & 0 \\
e & 0
\end{array}\right]\right\}\right) \quad(\text { by }(12)) \\
= & 4 p_{11}\left(\left\{\left\{\left[\begin{array}{ll}
0 & a \\
0 & 0
\end{array}\right]\left[\begin{array}{ll}
0 & b \\
0 & 0
\end{array}\right]\left[\begin{array}{ll}
c & 0 \\
0 & 0
\end{array}\right]\right\},\left[\begin{array}{ll}
0 & 0 \\
d & 0
\end{array}\right],\left[\begin{array}{ll}
0 & 0 \\
e & 0
\end{array}\right]\right\}\right) \\
& (\text { by }(14)) \\
= & 4 p_{11}(\{E D\{C B A\}\}) \quad(\text { by commutativity of the triple product }) \\
= & 4 p_{11}(\{C B\{E D A\}\})+4 p_{11}(\{\{E D C\} B A\}) \\
& -4 p_{11}(\{C\{B E D\} A\}) \quad(\text { by }(2)) \\
= & 0+4 p_{11}\left(\left\{\left\{\left[\begin{array}{ll}
0 & 0 \\
e & 0
\end{array}\right]\left[\begin{array}{cc}
0 & 0 \\
d & 0
\end{array}\right]\left[\begin{array}{ll}
c & 0 \\
0 & 0
\end{array}\right]\right\},\left[\begin{array}{cc}
0 & b \\
0 & 0
\end{array}\right],\left[\begin{array}{cc}
0 & a \\
0 & 0
\end{array}\right]\right\}\right)+0 \\
= & 2 p_{11}\left(\left\{\left[\begin{array}{ccc}
0 & a \\
0 & 0
\end{array}\right]\left[\begin{array}{ll}
0 & b \\
0 & 0
\end{array}\right]\left[\begin{array}{cc}
(c d e) & 0 \\
0 & 0
\end{array}\right]\right\}\right)(\text { by }(15)) \\
= & {[a b(c d e)]=[a b[c d e]] . }
\end{aligned}
$$

To complete the proof of associativity, consider

$$
\begin{aligned}
& {[a[d c b] e]} \\
& =\langle a\langle d c b\rangle e\rangle \\
& =2 p_{11}\left(\left\{\left[\begin{array}{ll}
0 & a \\
0 & 0
\end{array}\right]\left[\begin{array}{cc}
0 & 0 \\
0 & \langle d c b\rangle
\end{array}\right]\left[\begin{array}{ll}
0 & 0 \\
e & 0
\end{array}\right]\right\}\right) \text { (by (13)) } \\
& =4 p_{11}\left(\left\{\left[\begin{array}{ll}
0 & a \\
0 & 0
\end{array}\right],\left\{\left[\begin{array}{ll}
0 & 0 \\
d & 0
\end{array}\right]\left[\begin{array}{ll}
c & 0 \\
0 & 0
\end{array}\right]\left[\begin{array}{ll}
0 & b \\
0 & 0
\end{array}\right]\right\},\left[\begin{array}{ll}
0 & 0 \\
e & 0
\end{array}\right]\right\}\right) \\
& \text { (by (16)) } \\
& =4 p_{11}(\{A\{D C B\} E\}) \\
& =4 p_{11}(\{\{A B C\} D E\})+4 p_{11}(\{E B\{A D C\}\})-4 p_{11}(\{C\{B A D\} E\}) \\
& \text { (by (4)) } \\
& =4 p_{11}(\{\{A B C\} D E\})(\text { since } A \perp D) \\
& =4 p_{11}\left(\left\{\left\{\left[\begin{array}{ll}
0 & a \\
0 & 0
\end{array}\right]\left[\begin{array}{ll}
0 & b \\
0 & 0
\end{array}\right]\left[\begin{array}{ll}
c & 0 \\
0 & 0
\end{array}\right]\right\},\left[\begin{array}{ll}
0 & 0 \\
d & 0
\end{array}\right],\left[\begin{array}{ll}
0 & 0 \\
e & 0
\end{array}\right]\right\}\right) \\
& =2 p_{11}\left(\left\{\left[\begin{array}{cc}
{[a b c]} & 0 \\
0 & 0
\end{array}\right]\left[\begin{array}{ll}
0 & 0 \\
d & 0
\end{array}\right]\left[\begin{array}{ll}
0 & 0 \\
e & 0
\end{array}\right]\right\}\right)=([a b c] d e)(\text { by }(15)) \\
& =[[a b c] d e] \text {. }
\end{aligned}
$$


Lemma 5.2. Let $A$ be an operator space such that $M_{2}(A)_{0}$ is a bounded symmetric domain, so that by Proposition 5.1, A is a $\mathrm{C}^{*}$-ternary ring. Suppose that the $\mathrm{C}^{*}$-ternary ring $A$ is isomorphic to a TRO, that is, $A_{-1}=0$ in Theorem 2.2. Form the ternary product $[\cdots]_{M_{2}(A)}$ induced by the ternary product on $A$ as if it was ordinary matrix multiplication, that is, if $X=$ $\left[x_{i j}\right], Y=\left[y_{k l}\right], Z=\left[z_{p q}\right] \in M_{2}(A)$, then $[X Y Z]_{M_{2}(A)}$ is the matrix whose $(i, j)$-entry is $\sum_{p, q}\left[x_{i p} y_{q p} z_{q j}\right]$. Then

$$
2\{X Y Z\}_{M_{2}(A)}=[X Y Z]_{M_{2}(A)}+[Z Y X]_{M_{2}(A)}
$$

Proof. It suffices to prove that $\{X X X\}_{M_{2}(A)}=[X X X]_{M_{2}(A)}$. In the first place,

$$
[X X X]_{M_{2}(A)}=\left[\begin{array}{c|c}
{\left[x_{11} x_{11} x_{11}\right]+\left[x_{12} x_{12} x_{11}\right]} & {\left[x_{11} x_{11} x_{12}\right]+\left[x_{12} x_{12} x_{12}\right]} \\
+\left[x_{11} x_{21} x_{21}\right]+\left[x_{12} x_{22} x_{21}\right] & +\left[x_{11} x_{21} x_{22}\right]+\left[x_{12} x_{22} x_{22}\right] \\
\hline\left[x_{21} x_{11} x_{11}\right]+\left[x_{22} x_{12} x_{11}\right] & {\left[x_{21} x_{11} x_{12}\right]+\left[x_{22} x_{12} x_{12}\right]} \\
+\left[x_{21} x_{21} x_{21}\right]+\left[x_{22} x_{22} x_{21}\right] & +\left[x_{21} x_{21} x_{22}\right]+\left[x_{22} x_{22} x_{22}\right]
\end{array}\right] .
$$

On the other hand, by using Lemmas 3.3, 3.4, 3.6 and 3.8, and Proposition 3.9 ,

$$
\begin{aligned}
& \sum_{k, l, p, q}\left\{P_{11}(X) P_{k l}(X) P_{p q}(X)\right\} \\
& =\left[\begin{array}{c|c}
\left\{x_{11} x_{11} x_{11}\right\}+\left[x_{12} x_{12} x_{11}\right] / 2 & {\left[x_{11} x_{11} x_{12}\right] / 2} \\
+\left[x_{11} x_{21} x_{21}\right] / 2 & +\left[x_{11} x_{21} x_{22}\right] / 2 \\
\hline\left[x_{21} x_{11} x_{11}\right] / 2+\left[x_{22} x_{12} x_{11}\right] / 2 & 0
\end{array}\right],
\end{aligned}
$$




$$
\begin{aligned}
& \sum_{k, l, p, q}\left\{P_{12}(X) P_{k l}(X) P_{p q}(X)\right\} \\
& =\left[\begin{array}{c|c}
{\left[x_{12} x_{22} x_{21}\right] / 2} & \left\{x_{12} x_{12} x_{12}\right\}+\left[x_{11} x_{11} x_{12}\right] / 2 \\
+\left[x_{12} x_{12} x_{11}\right] / 2 & +\left[x_{12} x_{22} x_{22}\right] / 2 \\
\hline 0 & {\left[x_{21} x_{11} x_{12}\right] / 2+\left[x_{22} x_{12} x_{12}\right] / 2}
\end{array}\right], \\
& \sum_{k, l, p, q}\left\{P_{21}(X) P_{k l}(X) P_{p q}(X)\right\} \\
& =\left[\begin{array}{c|c}
{\left[x_{11} x_{21} x_{21}\right] / 2+\left[x_{12} x_{22} x_{21}\right] / 2} & 0 \\
\hline\left\{x_{21} x_{21} x_{21}\right\}+\left[x_{21} x_{11} x_{11}\right] / 2 & {\left[x_{21} x_{11} x_{12}\right] / 2} \\
+\left[x_{22} x_{22} x_{21}\right] / 2 & +\left[x_{21} x_{21} x_{22}\right] / 2
\end{array}\right],
\end{aligned}
$$

and

$$
\begin{aligned}
& \sum_{k, l, p, q}\left\{P_{22}(X) P_{k l}(X) P_{p q}(X)\right\} \\
& =\left[\begin{array}{c|c}
0 & {\left[x_{11} x_{21} x_{22}\right] / 2+\left[x_{12} x_{22} x_{22}\right] / 2} \\
\left.\hline x_{22} x_{12} x_{11}\right] / 2 & \left\{x_{22} x_{22} x_{22}\right\}+\left[x_{21} x_{21} x_{22}\right] / 2 \\
+\left[x_{22} x_{22} x_{21}\right] / 2 & {\left[x_{22} x_{12} x_{12}\right] / 2}
\end{array}\right] .
\end{aligned}
$$

Since $\{X X X\}_{M_{2}(A)}=\sum_{i, j} \sum_{k, l, p, q}\left\{P_{i j}(X) P_{k l}(X) P_{p q}(X)\right\}$ and $\{x x x\}=$ $[x x x]$, the lemma follows.

We now state and prove the main result of this paper.

Theorem 5.3. Let $A \subset B(H)$ be an operator space and suppose that $M_{n}(A)_{0}$ is a bounded symmetric domain for some $n \geq 2$. Then $A$ is $n$-isometric to a ternary ring of operators (TRO). If $M_{n}(A)_{0}$ is a bounded symmetric domain 
for all $n \geq 2$, then $A$ is ternary isomorphic and completely isometric to a TRO.

Proof. The second statement follows from the first one. Suppose $n=2$. From Theorem 2.2 and Proposition 5.1, we know that $A=A_{1} \oplus A_{-1}$ where $A_{1}$ is ternary isomorphic to a TRO $B$ and $A_{-1}$ is anti-isomorphic to a TRO $C$. Let $\varphi: A_{-1} \rightarrow C$ be an anti-isomorphism. Since $C$ is a $\mathrm{JB}^{*}$-triple under the product $\left\{\begin{array}{lll}x & y & z\end{array}\right\}=(1 / 2)\left(x y^{*} z+z y^{*} x\right)$ and $\varphi$ is an isometry, hence a triple isomorphism, it follows that

$$
\varphi(x) \varphi(x)^{*} \varphi(x)=\varphi\{x x x\}=\varphi[x x x]=-\varphi(x) \varphi(x)^{*} \varphi(x)
$$

so that $\varphi(x) \varphi(x)^{*} \varphi(x)=0$ and $x=0$. Thus $A_{-1}=0$ and $A$ is ternary isomorphic to a TRO $B$. Let $\psi: A \rightarrow B$ be a surjective ternary isomorphism. Then by Lemma 5.2, the amplification $\psi_{2}$ is a triple isomorphism of the JB*triple $M_{2}(A)$ onto the $\mathrm{JB}^{*}$-triple $M_{2}(B)$, with the triple product

$$
\{R S T\}_{M_{2}(B)}:=\left(R S^{*} T+T S^{*} R\right) / 2,
$$

implying that $\psi_{2}$ is a triple isomorphism, hence an isometry. Thus, $A$ is 2isometric to $B$, proving the theorem for $n=2$. The general case for $M_{n}(A)$ is now not difficult to obtain. We require only one short lemma.

Lemma 5.4. Let $A$ be an operator space such that for some $n \geq 3, M_{n}(A)$ has a $\mathrm{JB}^{*}$-triple structure. Then for $X, Y, Z \in M_{n}(A)$, the following products all vanish:

- $\left\{P_{i j}(X) P_{k j}(Y) P_{l j}(Z)\right\}$ (for distinct $\left.i, k, l\right)$

- $\left\{P_{i j}(X) P_{i k}(Y) P_{i l}(Z)\right\}$ (for distinct $\left.j, k, l\right)$

- $\left\{P_{i j}(X) P_{k l}(Y) P_{p q}(Z)\right\}($ for $i \neq k, j \neq l$ and either $p \notin\{i, k\}$ or $q \notin\{j, l\})$.

Proof. Two applications of the fact that the range of a bicontractive projection on a JB*-triple is a subtriple yield that $\left\{P_{i j}(X) P_{k j}(Y) P_{l j}(Z)\right\}$ lies in $\left(P_{i j}+P_{k j}+P_{l j}\right) M_{n}(A)$. However, by a conditional expectation property,

$$
\left(P_{i j}+P_{k j}\right)\left\{P_{i j}(X) P_{k j}(Y) P_{l j}(Z)\right\}=\left(P_{i j}+P_{k j}\right)\left\{P_{i j}(X) P_{k j}(Y) 0\right\}=0 .
$$

A similar calculation shows $\left(P_{k j}+P_{l j}\right)\left\{P_{i j}(X) P_{k j}(Y) P_{l j}(Z)\right\}=0$, proving the first statement. A similar agrument proves the second statement. The proof of the last statement is the same as the proof of Lemma 3.3. For $n=3$, one needs to prove, for example, that

$$
D\left(\left[\begin{array}{lll}
a & 0 & 0 \\
0 & 0 & 0 \\
0 & 0 & 0
\end{array}\right],\left[\begin{array}{lll}
0 & 0 & 0 \\
0 & b & 0 \\
0 & 0 & 0
\end{array}\right]\right)=0 .
$$


Returning to the proof of Theorem 5.3, if $M_{n}(A)$ is a JB*-triple, then $M_{2}(A)$, which is isometric to the range of a contractive projection on $M_{n}(A)$, is also a $\mathrm{JB}^{*}$-triple. Hence, by the $n=2$ case, $A$ is a $\mathrm{C}^{*}$-ternary ring which is ternary isomorphic and isometric under a map $\phi$ to a TRO $B$ and $M_{2}(A)$ is triple isomorphic and isometric to $M_{2}(B)$ under the amplification $\phi_{2}$. Every triple product $\{X Y Z\}$ in $M_{n}(A)$ is the sum of products of the form $\left\{P_{i j}(X) P_{k l}(Y) P_{p q}(Z)\right\}$. By Lemma 5.4, every such product of matrix elements in $M_{n}(A)$ is either zero or takes place in the intersection of two rows with two columns. The subspace of $M_{n}(A)$ defined by one such intersection is a subtriple of $M_{n}(A)$ since it is the range of the product of two bicontractive projections. It is isometric, via

$$
P_{i j}(X)+P_{i l}(Y)+P_{k j}(Z)+P_{k l}(W) \mapsto\left[\begin{array}{cc}
P_{i j}(X) & P_{i l}(Y) \\
P_{k j}(Z) & P_{k l}(W)
\end{array}\right],
$$

hence triple isomorphic, to $M_{2}(A)$. Hence, by the proof of the $n=2$ case, all triple products in $M_{n}(A)$ are the natural ones obtained from the ternary structure on $A$ as in Lemma 5.2. It follows that $M_{n}(A)$ is triple isomorphic to $M_{n}(B)$ via the amplification map $\phi_{n}$ which is thus an isometry.

As application, we offer two corollaries.

Corollary 5.5. Let $\mathcal{A} \subset B(H, K)$ be a TRO and let $P$ be a completely contractive projection on $\mathcal{A}$. Then the range of $P$ is completely isometric to another TRO.

Proof. Since $\mathcal{A}$ is a TRO, $M_{n}(\mathcal{A})$ is a JB*-triple. Therefore $M_{n}(P(\mathcal{A}))=$ $P_{n}\left(M_{n}(\mathcal{A})\right)$ is a $\mathrm{JB}^{*}$-triple, and its unit ball is a bounded symmetric domain.

Another way to obtain this corollary is to note that every TRO is a corner of a $\mathrm{C}^{*}$-algebra and hence the range of a completely contractive projection on that algebra. By composing these two projections, the corollary is reduced to $[38]$.

Our second corollary is a variant of the fundamental Choi-Effros result.

Corollary 5.6. Let $P$ be a unital 2-positive projection on a unital $\mathrm{C}^{*}$ algebra $A$. Then $P(A)$ is 2-isometric to a $\mathrm{C}^{*}$-algebra. If $P$ is completely positive and unital, then $P(A)$ is completely isometric to a $\mathrm{C}^{*}$-algebra.

In order to state our second theorem, we recall that a complex Banach space $A$ is linearly isometric to a unital $\mathrm{JB}^{*}$-algebra if and only if its open unit ball $A_{0}$ is a bounded symmetric domain of tube type [11]. In [37], a necessary and sufficient condition, involving the Lie algebra of all complete holomorphic vector fields on $A_{0}$, is given for such $A$ to be obtained from a $\mathrm{C}^{*}$-algebra with the anticommutator product. Our next theorem gives a holomorphic characterization of $\mathrm{C}^{*}$-algebras up to complete isometry. 
Theorem 5.7. Let $A \subset B(H)$ be an operator space and suppose that $M_{n}(A)_{0}$ is a bounded symmetric domain for some $n \geq 2$. If the induced bounded symmetric domain structure on $A_{0}$ is of tube type, then $A$ is $n$-isometric to a $\mathrm{C}^{*}$-algebra. If $M_{n}(A)_{0}$ is a bounded symmetric domain for all $n \geq 2$ and $A_{0}$ is of tube type, then $A$ is completely isometric to a $\mathrm{C}^{*}$-algebra.

Proof. By Theorem 5.3, we may assume that $A$ is a TRO. Since $A$ has the structure of a unital JB*-algebra, there is a partial isometry $u \in A$ such that $a u^{*} u=u u^{*} a=a$ for every $a \in A$. Then $A$ becomes a $\mathrm{C}^{*}$ algebra with product $a \cdot b=a u^{*} b$ and involution $a^{\sharp}=u a^{*} u$. Since $a b^{*} c=$ $a \cdot b^{\sharp} \cdot c$, and ternary isomorphisms of TRO's are complete isometries, the result follows.

Remark 5.8. One can construct operator spaces that are 2-isometric to a $\mathrm{C}^{*}$-algebra $A$ which are not completely isometric to $A$. Hence, if $M_{2}(A)_{0}$ is a bounded symmetric domain it does not follow that $M_{n}(A)_{0}$ is a bounded symmetric domain for every $n \geq 2$. It would be interesting to see if this were true under some further condition on $A$. The proof of Theorem 5.3 seems to require a bounded symmetric domain structure on $M_{2}(A)_{0}$, not simply on $M_{1,2}(A)_{0}$ for example. It would be interesting to see what could be said if it is assumed that $M_{1, n}(A)_{0}$ were a bounded symmetric domain for every $n \geq 2$.

Acknowledgements. The authors wish to thank Profesors Zhong-Jin Ruan and David Blecher for their advice and encouragement at the beginning stages of this work.

\section{References}

[1] K. El Amin, A.M. Campoy and A.R. Palacios, A holomorphic characterization of $\mathrm{C}^{*}$ - and JB*-algebras, Manu. Math., 104 (2001), 467-478, MR 2002e:46085, Zbl 0982.46040 .

[2] J. Arazy and Y. Friedman, Contractive projections in $C_{1}$ and $C_{\infty}$, Mem. Amer. Math. Soc., 13(200), 1978, MR 82b:47023, Zbl 0382.47020.

[3] W.B. Arveson, Subalgebras of $\mathrm{C}^{*}$-algebras, Acta Math., 123 (1969), 141-224, MR 40 \#6274, Zbl 0194.15701.

[4] , Subalgebras of $\mathrm{C}^{*}$-algebras II, Acta Math., 128 (1972), 271-308, MR 52 \#15035, Zbl 0245.46098.

[5] T.J. Barton and R. Timoney, On biduals, preduals and ideals of JB*-triples, Math. Scand., 59 (1986), 177-191.

[6] D. Blecher, The standard dual of an operator space, Pacific J. Math., 153 (1992), 15-30, MR 93d:47083, Zbl 0726.47030.

[7] _ A completely bounded characterization of operator algebras, Math. Ann., 303 (1995), 227-239, MR 96k:46098, Zbl 0892.47048.

[8] - The Shilov boundary of an operator space and the characterization theorems, J. Funct. Anal., 182 (2001), 280-343, MR 2002d:46049. 
[9] D. Blecher and V. Paulsen, Multipliers of operator spaces and the injective envelope, Pacific J. Math., 200 (2001), 1-17, MR 2002k:46150.

[10] D. Blecher, Z.-J. Ruan and A. Sinclair, A characterization of operator algebras, J. Funct. Anal., 89 (1990), 188-201, MR 91b:47098, Zbl 0714.46043.

[11] R. Braun, W. Kaup and H. Upmeier, A holomorphic characterization of Jordan $\mathrm{C}^{*}$ algebras, Math. Zeit., 161 (1978), 277-290, MR 58 \#12398, Zbl 0385.32002.

[12] M.-D. Choi and E. Effros, Injectivity and operator spaces, J. Funct. Anal., 24 (1977), 156-209, MR 55 \#3814, Zbl 0341.46049.

[13] C-H. Chu, M. Neal and B. Russo, Normal contractive projections preserve type. Preprint, 2001.

[14] S. Dineen, The second dual of a JB*-triple system, in 'Complex Analysis, Functional Analysis and Approximation Theory', J. Mujica (ed.), Amsterdam: Elsevier (North Holland), 1986, 67-69, MR 88f:46097, Zbl 0653.46053.

[15] E. Effros, N. Ozawa and Z.-J. Ruan, On injectivity and nuclearity for operator spaces, Duke Math. J., 110 (2001), 489-521, MR 2002k:46151.

[16] E. Effros and Z.J. Ruan, Operator Spaces, Oxford University Press, 2000, MR 2002a:46082, Zbl 0969.46002.

[17] E. Effros and E. Stormer, Positive projections and Jordan structure in operator algebras, Math. Scand., 45 (1979), 127-138, MR 82e:46076, Zbl 0455.46059.

[18] Y. Friedman and B. Russo, Contractive projections on $C_{0}(K)$, Trans. Amer. Math. Soc., 273 (1982), 57-73, MR 83i:46062, Zbl 0534.46037.

[19] _ Function representation of commutative operator triple systems, J. Lon. Math. Soc. (2), 27 (1983), 513-524, MR 84h:46095, Zbl 0543.46046.

[20] _ Conditional expectation without order, Pacific J. Math., 115 (1984), 351-360, MR 86b:46116, Zbl 0563.46039.

[21] Solution of the contractive projection problem, J. Funct. Anal., 60 (1985), 56-79, MR 87a:46115, Zbl 0558.46035.

[22] _ Structure of the predual of a $\mathrm{JBW}^{*}$-triple, J. Reine Angew. Math., 356 (1985), 67-89, MR 86f:46073, Zbl 0547.46049.

[23] M. Hamana, Injective envelopes of operator systems, Publ. Res. Inst. Math. Sci. Kyoto Univ., 15 (1979), 773-785, MR 81h:46071, Zbl 0436.46046.

[24] Triple envelopes and Šlov boundaries of operator spaces, Math. J. Toyama Univ., 22 (1999), 77-93, MR 2001a:46057.

[25] L.A. Harris, Bounded symmetric domains in infinite dimensional spaces, in 'Infinite Dimensional Holomorphy' (T.L. Hayden and T.J. Suffridge, eds.), Proceedings, 1973, Lecture Notes in Mathematics, 364, 13-40, Springer, 1974, MR 53 \#11106, Zbl 0293.46049.

[26] G. Horn, Classification of JBW*-triples of Type I, Math. Zeit., 196 (1987), 271-291, MR 88m:46076, Zbl 0615.46045.

[27] G. Horn and E. Neher, Classification of continuous JBW*-triples, Trans. Amer. Math. Soc., 306 (1988), 553-578, MR 89c:46090, Zbl 0659.46063.

[28] W. Kaup, A Riemann mapping theorem for bounded symmetric domains in complex Banach spaces, Math. Zeit., 183 (1983), 503-529, MR 85c:46040, Zbl 0519.32024.

[29] Contractive projections on Jordan $\mathrm{C}^{*}$-algebras and generalizations, Math. Scand., 54 (1984), 95-100, MR 85h:17012, Zbl 0578.46066. 
[30] Y. Lindenstrauss and D. Wulbert, On the classification of Banach spaces whose duals are $L_{1}$-spaces, J. Funct. Anal., 4 (1969), 332-349, MR 40 \#3274, Zbl 0184.15102.

[31] O. Loos, Bounded symmetric domains and Jordan pairs, Lecture Notes, Univ. of California, Irvine, 1977.

[32] M. Neal and B. Russo, Contractive projections and operator spaces, C.R. Acad. Sci. Paris, 331 (2000), 873-878, MR 2001m:46128, Zbl 0973.47052.

[33] _ Contractive projections and operator spaces, Trans. Amer. Math. Soc. (to appear).

[34] Z.-J. Ruan, Subspaces of $\mathrm{C}^{*}$-algebras, J. Funct. Anal., 76 (1988), 217-230, MR 89h:46082, Zbl 0646.46055.

[35] _ Injectivity of operator spaces, Trans. Amer. Math. Soc., 315 (1989), 89-104, MR 91d:46078, Zbl 0669.46029.

[36] B. Russo, Structure of JB*-triples, in 'Jordan Algebras', Proc. Oberwolfach Conference 1992 (W. Kaup, K. McCrimmon, H. Petersson, Eds.), de Gruyter, Berlin, 1994, 209-280, MR 95h:46109, Zbl 0805.46072.

[37] H. Upmeier, A holomorphic characterization of $\mathrm{C}^{*}$-algebras, in 'Functional Analysis, holomorphy and approximation theory, II' (Rio de Janeiro, 1981), North Holland Math. Stud., 86 (1984), 427-467, MR 86k:46089, Zbl 0546.46050.

[38] M.A. Youngson, Completely contractive projections on $\mathrm{C}^{*}$-algebras, Quart. J. Math., 34 (1983), 507-511, MR 85f:46112, Zbl 0542.46029.

[39] H. Zettl, A characterization of ternary rings of operators, Adv. Math., 48 (1983), 117-143, MR 84h:46093, Zbl 0517.46049.

Received November 22, 2001 and revised April 25, 2002. Both authors acknowledge the support of NSF Grant DMS-0101153.

Department of Mathematics

DENISON UNIVERSITY

Granville, OH 43023

E-mail address: nealm@denison.edu

Department of Mathematics

UNIVERSITY OF CALIFORNIA

IRVINE, CA 92697-3875

E-mail address: brusso@math.uci.edu 